\title{
Stochastic and nonlinear forcing of wavepackets in a Mach 0.9 jet
}

\author{
Aaron Towne*, Tim Coloniusł Peter Jordan $\ddagger$ André Cavalierił; Guillaume A. Brès
}

\begin{abstract}
Recent studies have shown that while linear wavepacket models accurately reproduce experimentally observed, low azimuthal-wavenumber pressure fluctuations in the near field of turbulent jets, they significantly under-predict the intensity of the acoustic radiation produced in the subsonic case. In a linear context, "jittering" of the wavepackets, which can arise due to both stochastic and nonlinear interactions that force the wavepackets, has been hypothesized as a mechanism by which the radiation efficiency of wavepackets is greatly increased. We use data from a carefully validated large-eddy-simulation of a Mach 0.9 turbulent jet to explore this hypothesis. We analyze the LES data in frequency space using windowed segments of a set of snapshots spanning two thousand acoustic time units. We apply the linearized Navier-Stokes operator to this data in order to compute the nonlinear forcing field that occurred in the LES simulations, and propose several techniques for educing the relation between the forcing and the observed flow fields. In particular, we employ empirical techniques to identify high energy modes (via proper orthogonal decomposition) in both the flow and acoustic fields, as well as a set of empirical resolvent modes that maximize either the gain between the forcing and flow fields, or the gain between the forcing and acoustic fields. The high gain modes are similar to the high energy modes in both cases, suggesting that the forcing fields are nearly uncorrelated in each realization. Both flow and acoustic fields appear to be driven by largely incoherent forcing corresponding to turbulence in the region of strong shear and, in particular, close to the critical layer. With the caveat that we have thus far only analyzed the axisymmetric mode of the disturbance fields, the results suggest that accurate linear wavepacket models that capture both the coherent flow and acoustic fields can be constructed if appropriate parameterizations of the stochastic forcing can be found, i.e. such forcings will excite the high gain modes to produce the observed coherent structures in both the near and far field.
\end{abstract}

\section{Introduction}

The relevance of large-scale coherent structures within the turbulent flow-field to the dominant, low frequency aft-angle sound propagation has long been established. ${ }^{1}$ The spatiotemporal signatures of these structures take the form of advecting wavepackets with slowly-varying wavelength and phase-speed. The emphasis has been on the modes that are well azimuthally correlated and represented by the first few wave numbers of an azimuthal Fourier transform. These first few azimuthal modes dominate the acoustic field of turbulent jets and have been directly correlated with the near-field wavepacket activity. ${ }^{1}$ This suggests that reduced-order jet-noise models could be constructed by modeling these wavepackets.

The typical approach to modeling wavepackets is to approximate them as linear modal solutions of the Euler or Navier-Stokes equations linearized about the long-time mean of the turbulent flow field. This approach dates to the 1970s, when it was found that certain properties of wavepackets in jets could be explained by locally paralle ${ }^{2,3}$ and weakly nonparalle ${ }^{4,5}$ linear stability theory. More recently, modal solutions have been obtained directly by solving parabolized stability equations (PSE), ${ }^{6,7}$ a global eigenvalue problem, ${ }^{8}$ or a boundary value problem with specified inlet perturbations. ${ }^{9}$

*Ph.D. student, California Institute of Technology, Pasadena, CA, USA

$\dagger$ Professor, California Institute of Technology, Pasadena, CA, USA

${ }^{\ddagger}$ Research Scientist, Institut Pprime, CNRS - University of Poitiers - ENSMA, Poitiers, France

$\S$ Assistant Professor, Instituto Tecnolõgico de Aeronáutica, São José dos Campos, Brazil

『 Senior Research Scientist, CASCADE Technologies, Palo Alto, CA, USA 
The near-field wavepackets obtained from these modal solutions show compelling agreement with those educed from experimental and simulation data for both subsonic and supersonic jets. ${ }^{6,7}$ For supersonic jets, these modal solutions also contain, with reasonable accuracy, the corresponding far-field acoustic radiation. ${ }^{7}$ On the other hand, linear modal solutions for subsonic jets have been shown to under-predict far-field acoustic radiation by as much as $40 \mathrm{~dB}$ despite the close match in the near-field wavepacket. ${ }^{9,10}$ The source of the discrepancy has been hypothesized to be "jitter" of the wavepackets caused by one of several mechanisms that can be associated with stochastic forcing of the wavepackets. The idea is that while the linear models can predict reasonably the "typical" near-field wavepacket, small differences (jitter) in different instantaneous realizations of the wavepacket will amplify its radiative efficiency. That is to say, the typical wavepacket does not make the typical sound, and we must also predict the coherence (or lack thereof) of the wavepacket to properly capture the observed sound levels. ${ }^{11-13}$

In this paper, we use data from a carefully validated large-eddy-simulation of a Mach 0.9 turbulent jet to explore how actual wavepackets are forced by nonlinear interactions. Consider the Navier-Stokes equations,

$$
\frac{\partial q}{\partial t}=\mathcal{N}(q, q)+g(x, r, \theta, t)
$$

where $\mathcal{N}(q, q)$ are the standard terms representing the inviscid fluxes and viscous terms (not written here for brevity), and $g$ is a zero-mean stochastic forcing term to be discussed shortly. Note that $\mathcal{N}$ is a bilinear operator and $q$ is the vector of flow variables, $q=\left[u_{x}, u_{r}, u_{\theta}, \nu, p\right]^{T}, \nu=\frac{1}{\rho}$. We add the stochastic forcing $g$ to represent the reality that in simulations and experiments of stationary, turbulent jets, there is always stochastic forcing. The forcing is organic in the case of experiments, consisting of environmental fluctuations, turbulence inside the nozzle feeding the jet, and so on. In the case of simulations, the forcing is artificially added, through randomized inlet or nozzle boundary layer fluctuations, to try to reproduce realistic turbulence.

Substituting the standard Reynolds decomposition $q=\bar{q}(x, r)+q^{\prime}(x, r, \theta, t)$ into equation (1) gives

$$
\frac{\partial q^{\prime}}{\partial t}-\mathcal{N}\left(\bar{q}, q^{\prime}\right)-\mathcal{N}\left(q^{\prime}, \bar{q}\right)=\mathcal{N}(\bar{q}, \bar{q})+\mathcal{N}\left(q^{\prime}, q^{\prime}\right)+g .
$$

The left-hand-side of equation (2) can be identified as the linearization of the Navier-Stokes operator about $\bar{q}$. With the right-hand-side neglected, this equation is the starting point for linear wavepacket models. The first right-hand-side term is a zero-frequency correction term that is nonzero whenever $\bar{q}$ is not a fixed point of $\mathcal{N}$. If the flow is statistically stationary, then $\mathcal{N}(\bar{q}, \bar{q})=-\overline{\mathcal{N}\left(q^{\prime}, q^{\prime}\right)}$, which expresses the RANS equations for the mean. The second right-hand-side term represents nonlinear interactions of the fluctuations $q^{\prime}$.

Instead of neglecting the two right-hand-side terms, we interpret them as a volumetric forcing of the linear system, and write

$$
\frac{\partial q^{\prime}}{\partial t}=\mathcal{A}(\bar{q}) q^{\prime}+f(x, r, \theta, t)
$$

where $\mathcal{A}(\bar{q}) q^{\prime}=\mathcal{N}\left(\bar{q}, q^{\prime}\right)+\mathcal{N}\left(q^{\prime}, \bar{q}\right)$ and $f(x, r, \theta, t)=\mathcal{N}(\bar{q}, \bar{q})+\mathcal{N}\left(q^{\prime}, q^{\prime}\right)+g$. Note that no approximation has been made to this point, and equation (3) is exact if the proper forcing field $f$ is known.

Since we are interested in an averaged description of the process, we consider an ensemble of solutions to equation (3), each forced by an independent realization of the random forcing $g$. The $k$-th realization (denoted $q^{(k)}$ ) is then governed by

$$
\frac{\partial q^{\prime(k)}}{\partial t}=\mathcal{A}(\bar{q}) q^{\prime(k)}+f^{(k)}(x, r, \theta, t)
$$

Since our interest is in stationary, round jets, it suffices to decompose each realization $q^{\prime(k)}$ into azimuthal and temporal Fourier modes:

$$
q^{\prime(k)}(x, r, \theta, t)=\sum_{n} \sum_{m} \hat{q}_{\omega, m}^{(k)} e^{\imath m \theta} e^{\imath \omega_{n} t} .
$$

Each mode $\hat{q}_{\omega, m}^{(k)}$ then satisfies an equation of the form

$$
\mathcal{M}_{\omega, m} \hat{q}_{\omega, m}^{(k)}=\hat{f}_{\omega, m}^{(k)}
$$

where $\hat{f}_{\omega, m}$ is the appropriate Fourier component of the forcing term, $\mathcal{M}_{\omega, m}=\imath \omega I-\mathcal{A}_{m}$, and $\mathcal{A}_{m}$ is the operator $\mathcal{A}$ with $\frac{\partial}{\partial \theta}$ replaced by $\imath m$. 
In our prior wavepacket models, ${ }^{6,7}$ the entire right-hand-side of equation (6) is neglected and wavepackets are instead excited through boundary conditions enforced just downstream of the nozzle inlet. The role of turbulence in these models was therefore relegated to its role in establishing the mean flow field; it does not directly force the wavepackets. The inlet boundary condition is an artifact of having incomplete information about the near-nozzle flow field, and for PSE models in particular, the boundary condition takes the form of imposing the most spatially amplified solution of the associated parallel flow operator for the mean flow at the inlet. As nonlinearity is neglected and PSE tracks only the dominant spatial wavelength at any frequency, the statistical effects of any imposed random fluctuations (for example setting the amplitude or phase of the inlet disturbances to random values), is simply propagated through the system without meaningfully modifying the wavepackets or their acoustic efficiency. As was mentioned above, these models reproduce the observed near-field wavepackets reasonably well, but vastly under-predict their associated acoustic radiation.

In preliminary analysis of the present LES database, one specific hypothesis for the source of wavepacket amplification was analyzed, ${ }^{10}$ namely the effect of slow-time variation of the turbulent mean flow field. This was analyzed in two distinct ways: time-domain linearized Euler equations were solved using an unsteady base flow found by lowpass-filtering the LES fields, and frequency-domain solutions of equation (6) were found using PSE but with different short-duration averages of the mean flow field $\bar{q}$ used in defining $\mathcal{M}$. The idea was to account for intermittency observed in both LES and experimental wavepackets on a time scale much longer than the energetic wavepackets, corresponding to jet Strouhal numbers of $S t<0.05$. The results of this analysis showed that accounting for intermittency in this way increased the acoustic output of the wavepackets by as much as $20 \mathrm{~dB}$ at peak aft angles. But there remained a large discrepancy, again as much as $20 \mathrm{~dB}$, between the models and the directly computed acoustic field from LES.

The goal of the present analysis is to generalize these previous studies using LES data to educe the actual forcing experienced by wavepackets, which is bound up in the function $\hat{f}^{(k)}$. To do this, we collect LES data for $\hat{q}^{(k)}$ and apply to it the linearized operator, $\mathcal{M}$ to obtain corresponding forces, $\hat{f}^{(k)}$. We then utilize two modal decompositions, one of which is novel, to highlight different aspects of the relationship between $\hat{f}^{(k)}$ and $\hat{q}^{(k)}$.

At this stage of the analysis, we limit our attention to axisymmetric $(m=0)$ solutions of equation (6). The axisymmetric mode is important in the aft acoustic field, comprising roughly half of the emitted sound at the peak angles and frequencies of emission. In future work, we will return to the data to examine the results for $m=1$ and $m=2$.

The remainder of the paper is organized as follows. In the next section, we describe the LES database, its validation, and our post-processing routines for computing the forces $\hat{f}^{(k)}$. Next, in section III we recast equation (6) as an input-output system, and derive equations for several modal decompositions that are then used to investigate and explain the role of the nonlinear and stochastic forcing in the observed coherent structures in both the flow and acoustic fields. Results are given in section IV, followed by a brief summary of observations and some suggestions for further work in section V.

\section{LES database}

We use data from a high-fidelity large eddy simulation (LES) of an isothermal Mach 0.9 jet issued from a contoured convergent-straight nozzle. ${ }^{14,15}$ The accuracy of the LES data was verified via extensive comparisons with measurements taken from a companion experiment conducted at the PPRIME Institute, Poitiers. ${ }^{15}$ For the purpose of analysis, the LES data has been interpolated from the original unstructured LES grid onto a structured cylindrical grid that extends thirty jet diameters in the axial direction and six jet diameters in the radial direction. The grid spacings in both directions are nonuniform and were chosen to approximately correspond to the underlying LES resolution, with a total of 656 points in $x$ and 138 points in $r$. The azimuthal grid consists of 128 uniformly spaced angles. The database consists of time series, sampled at an interval of 0.2 acoustic time units, for the primitive flow variables on this grid. The computational setup and a sample flow field from the LES are shown in Figure 1.

To create an ensemble of statistically independent realizations of the jet, we partition the LES timeseries into 312 blocks with $50 \%$ overlap. Each block contains 64 instantaneous snapshots of the jet, resulting in a period corresponding to a Strouhal number of 0.087. We have also constructed ensembles based on blocks with half this period $(S t=0.043)$ and found little difference in the final results of the paper. The frequency content (at integer multiples of the period) are calculated for each block by taking a discrete Fourier transform in the azimuthal direction and time, resulting in an ensemble of jet realizations at each 


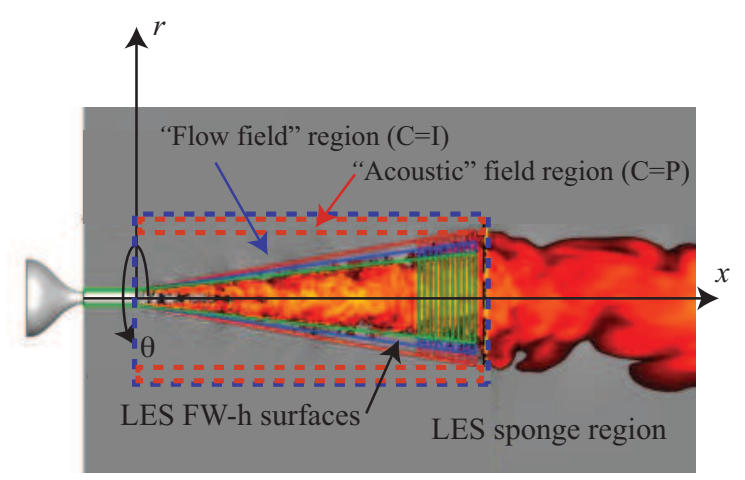

Figure 1: Schematic of the $M=0.9$ isothermal jet.

resolved frequency-azimuthal wavenumber pair.

We next calculate the nonlinear forcing terms $f_{\omega, m}^{(k)}$. These are the forces that would need to be applied to the linear model given by equation (6) to generate the LES data. Instead of computing the Fourier transforms of the many nonlinear products involved in the definition of $f^{(k)}$, we take advantage of the equality of equation (6) and instead evaluate its left-hand-side, which only requires the Fourier transform of $q^{\prime}$ and not its nonlinear products.

Applying the operator $\mathcal{M}_{\omega, m}$ to the LES data requires the computation of spatial derivatives of the data. Because we include viscous terms, both first and second derivatives are required. The most consistent approach to obtaining these derivatives would be to compute them on the LES grid using the native LES differentiation operators and interpolating them onto the same structured grid used for the data. This data was not, however, computed at the time the database was established and its inclusion would vastly increase the size of the database.

Instead, the derivatives are obtained by fitting the data with third-order smoothing splines ${ }^{16}$ and differentiating the splines. We checked the accuracy of these derivatives in several ways. First, we compared them to those obtained based on the LES differentiation for one temporal snapshot. The difference between the two sets of derivatives was found to be small over most of the domain, with the largest discrepancies along the lip-line very near the nozzle. We also computed the derivatives obtained from splines that were fit to only one-fourth of the total data points. While there were again minor differences in the smaller scales, particularly in the resulting forces, none of the changes were significant enough to change the results we present in this paper. This suggests that the derivatives, and therefore the forces, are sufficiently converged such that the results are dominated by physical phenomena rather than numerical noise.

\section{Modal decompositions}

In this section we describe several modal decompositions that have been applied to the LES data $\hat{q}^{(k)}$ and forces $\hat{f}^{(k)}$ in order to educe the nonlinear forces associated with hydrodynamically and acoustically important coherent structures in the jet.

\section{III.A. Preliminaries}

In simulations and models, the partial differential equations above are discretized in space, resulting in a system of ODEs in the time domain and algebraic equations in the frequency domain. For notational simplicity in what follows, we use the same symbols to denote the flow variables and their discrete representation on the grid. For example, $q^{\prime}$ and $\hat{q}$ become vectors of the five flow variables at each grid point, and the linear operators $\mathcal{A}$ and $\mathcal{M}$ become matrices, $A$ and $M=\imath \omega I-A$, respectively. The specifics of the discretization of these operators are implied by the previously described method of calculating the forces. This permits a convenient matrix notation for the data processing. Where it will not cause ambiguity, we also drop the subscripts $(\omega, m)$. 
It is helpful to write equation (6) as an input-output system:

$$
\begin{gathered}
M \hat{q}^{(k)}=B \hat{u}^{(k)} \\
\hat{y}^{(k)}=C \hat{q}^{(k)}
\end{gathered}
$$

where $\hat{u}^{(k)}$ are inputs, $\hat{y}^{(k)}$ are outputs, and $B$ and $C$ are (not necessarily square) matrices that can in general be functions of position in space, the mean flow field, frequency, and/or azimuthal wavenumber.

In linear dynamical systems, $\hat{u}^{(k)}$ represents a control input, such as the voltage to an actuator. In the present context, we interpret $\hat{u}^{(k)}$ as the full nonlinear (and stochastic) forcing term, $\hat{f}^{(k)}$, and take $B=I$. Thus we allow the forcing to excite each degree of freedom of the system.

We will consider three different choices for the output (i.e. different choices of $C$ ). The first is simply $C=I$, meaning the entire flow field over $0<x / D<30,0<r / D<6$ is included in the output. The second, which we label $C=P$, is a constant matrix with zeros in the entries corresponding to all variables except pressure, and at all points in space except a portion of the acoustic field, namely the sub region $0<x / D<30$ and $5<r / D<6$. These choices are shown in the schematic diagram in Figure 1 . The third choice is to set $C=M$, which allows us to consider the right-hand-side forcing field as the output, as expanded upon below.

We note that the acoustic field we are considering is not the asymptotic far-field, though the pressure field is entirely propagative at these values of $r$ for all the frequencies considered here, and the directivity is close to that of the far-field. We tried defining the region comprising the acoustic output as different subsets of the selected region with minimal impact on the presented results.

The ensembles of LES data described in Section II provide $N_{s}=312$ realizations of the system. Specifically the frequency-azimuthal wavenumber ensembles, and the corresponding forces, represent $N_{s}$ solutions of equation (7a), and the acoustic output of each is given by equation (7b). As is typical in modal analysis, this can be compactly denoted by forming a matrix whose columns represent the different realizations,

$$
Q=\left[\begin{array}{llll}
\hat{q}^{(1)} & \hat{q}^{(2)} & \cdots & \hat{q}^{\left(N_{s}\right)}
\end{array}\right], \quad F=\left[\begin{array}{llll}
\hat{f}^{(1)} & \hat{f}^{(2)} & \cdots & \hat{f}^{\left(N_{s}\right)}
\end{array}\right], \quad Y=\left[\begin{array}{llll}
\hat{y}^{(1)} & \hat{y}^{(2)} & \cdots & \hat{y}^{\left(N_{s}\right)}
\end{array}\right] .
$$

Since each realization is a solution, we have

$$
\begin{aligned}
& M Q=F, \\
& Y=C Q
\end{aligned}
$$

The modal decompositions we consider construct modes as linear combinations of the data:

$$
\tilde{q}_{j}=\sum_{k=1}^{N_{s}} \hat{q}^{(k)} \psi_{k j}
$$

which can be written in matrix form as

$$
\tilde{Q}=Q \Psi
$$

where the modes are columns of $\tilde{Q}$, ordered in some natural way. The goal of each decomposition is to determine the coefficient matrix $\Psi$ that produces modes that optimally satisfy an objective. The coefficient matrix also defines the output and forcing that correspond to each mode:

$$
\begin{aligned}
& \tilde{F}=F \Psi, \\
& \tilde{Y}=Y \Psi .
\end{aligned}
$$

By right-multiplying equation (9) by $\Psi$, it is clear that $\tilde{Q}, \tilde{F}$, and $\tilde{Y}$ together satisfy the linear system.

It is implicitly assumed for all of the decompositions that a large enough number of realizations of the dynamics $\left(N_{s}\right)$ have been incorporated such that the dominant modes are converged. We have verified the statistical convergence of our results by confirming that reducing the number of realizations by as much as $20 \%$ has negligible impact on the dominant modes. 


\section{III.B. Proper orthogonal decomposition}

The most common technique of extracting modes from empirical data is principle component analysis, also called the proper orthogonal decomposition (POD). ${ }^{17,18}$ POD constructs orthogonal modes from linear combinations of statistically independent data samples that optimally represent the data in terms of energy content. Specifically, reconstruction of the data using POD modes capture the largest possible portion of the total energy using any given number of modes, as measured by some suitable norm (or semi-norm)

$$
\|a\|=a^{*} W a \text {. }
$$

In the context of application to a discrete linear system, POD is entirely equivalent to principle component analysis (weighted by $W$ ); that is, the POD decomposes a set of statistically independent data samples into mutually incoherent (orthogonal) modes representing the principle components of the variance of the data set. The first POD mode is a coherent structure that accounts for a maximal portion of the variance of the data, and subsequent modes represent smaller and smaller portions of the variance.

The coefficient matrix that produces these POD modes satisfies the eigenvalue problem

$$
\Psi_{P O D}^{*} Q^{*} W Q \Psi_{P O D}=\Lambda_{P O D}
$$

The energy of each mode is given by the corresponding eigenvalue, found within the diagonal matrix $\Lambda$. The energy captured by a reduced-order reconstruction of the first $N_{r o}$ modes is found by summing the first $N_{\text {ro }}$ eigenvalues.

The meaning of the POD modes depends critically on the properties of the norm, in particular the weighting matrix, $W$. We choose to use the (semi)-norm induced by the output mapping, $W=C^{*} C$. This is sometimes referred to as extended $P O D .{ }^{19,20}$ For the case $C=I$, where the output is the entire flow field, this is equivalent to the standard Euclidean norm. For $C=P$, we are restricting attention to fluctuations that are correlated in the acoustic field. Finally, with $C=M$, we are essentially doing standard POD but with the forcing fields, $F$, replacing the states, $Q$. These modes are optimal for describing the energy of the forcing, F. Finally, other choices of weighting have been proposed; for example in balanced $\mathrm{POD}^{21}$ the observability Gramian is used for $W$.

\section{III.C. Empirical resolvent modes}

Resolvent modes are used to study the input/output characteristics of stationary, random processes. Specifically, resolvent modes maximize the gain between the input $\hat{u}$ and output $\hat{y}$ of such systems, where the gain is defined as

$$
\text { gain }=\frac{\|\hat{y}\|}{\|\hat{u}\|} \text {. }
$$

For linear time invariant systems such as equation (7), these solutions can be obtained at each frequency $\omega$ by computing the singular value decomposition of the transfer function $G(\omega)=C M^{-1} B$. Note that in the present case, $B=I$ and $\hat{u}=\hat{f}$. The singular values give the optimal gains and the right and left singular vectors define corresponding orthonormal inputs and responses, respectively.

Here, we formulate a method for calculating empirical resolvent modes (ERM). Specifically, we seek modes in the form of equation (11) that maximize the gain for every mode. In terms of the coefficient matrix, we want

$$
\Psi_{E R M}=\underset{\Psi}{\arg \max } \frac{\|\tilde{Y}\|}{\|\tilde{F}\|}=\underset{\Psi}{\arg \max } \frac{\Psi^{*} Y^{*} Y \Psi}{\Psi^{*} F^{*} F \Psi} .
$$

The optimal coefficient matrix is obtained by simultaneous diagonalization of the output and forcing matrices:

$$
\begin{gathered}
\tilde{Y}^{*} \tilde{Y}=\Psi_{E R M}^{*} Q^{*} C^{*} C Q \Psi_{E R M}=\Lambda_{E R M}, \\
\tilde{F}^{*} \tilde{F}=\Psi_{E R M}^{*} F^{*} F \Psi_{E R M}=I .
\end{gathered}
$$

The existence of such a $\Psi$ is guaranteed by a standard result of linear algebra: two Hermitian matrices can always be simultaneously diagonalized by a congruence transformation. ${ }^{22}$ In this case, $Y^{*} Y$ and $F^{*} F$ are the Hermitian matrices and $\Psi$ is the congruence transformation. The $\Psi$ that achieves this can be computed 
using a two-step process. First, the eigenvalue decomposition of $F^{*} F$ is computed. This is identical to computing the POD modes of $F$ :

$$
\Psi_{P O D-F}^{*} F^{*} F \Psi_{P O D-F}=\Lambda_{P O D-F}
$$

Second, the following eigenvalue decomposition is computed:

$$
V^{*}\left(\Lambda_{P O D-F}^{-1 / 2}\right)^{*} \Psi_{P O D-F}^{*} Y^{*} Y \Psi_{P O D-F} \Lambda_{P O D-F}^{-1 / 2} V=\Lambda_{E R M} .
$$

Then the ERM coefficient matrix is given by $\Psi_{E R M}=\Psi_{P O D-F} \Lambda_{P O D-F}^{-1 / 2} V$. The first step ensures that the ERM forces are orthonormal, while the second step ensures that the outputs are orthogonal, which maximizes the gains, which are given by $\Lambda_{E R M}$. Thus, the ERM modes can be computed by two eigendecompositions of symmetric, positive semi-definite matrices of size $N_{s} \times N_{s}$.

In the limit in which $F$ is full-rank, every possible mode of the system is excited, and the ERM modes exactly recover those obtained by direct computation of the singular value decomposition of $G(\omega)$. When $F$ is not full rank, which will always be the case in application, both the forcing and responses are constrained to lie within the spaces spanned by the data, and the ERM finds the modes with optimal gain under this constraint. Our expectation is that the high-gain modes should have a presence in the LES data, since they are by definition easily excited. However, it should be kept in mind that the turbulent fluctuations being convolved into $\hat{f}$ (from all frequencies and azimuthal modes) are, in the LES data, constrained to a turbulent equilibrium such that the overall flow is stationary. It is unclear therefore how well or quickly the ERM modes do converge to the high gain modes computed directly from SVD of $G(\omega)$. In the future we intend to make such comparisons.

Finally, note that if the different realizations of the forces are uncorrelated, then $F$ is orthogonal and ERM modes are equivalent to POD modes. This can be clearly seen by comparing equation (17) to equation (14). Therefore, any difference between POD and ERM modes are due to some bias in the forcing, leading to preferential excitation of certain modes.

\section{III.D. Remarks}

POD and related techniques have been applied broadly in fluid mechanics, and in particular to the flow fields ${ }^{23-25}$ and acoustic fields ${ }^{26}$ of jets. The results of these analyses are summarized in a recent review. ${ }^{1}$

Resolvent modes have been computed directly from the operator $G(\omega)$ to study the input/output behavior of both supersonic and subsonic jets, ${ }^{27,28}$ but, to our knowledge, the use of empirical data to construct them (for any flow) via the ERM is novel.

The ERM decomposition bears certain similarities with the Observer Inferred Decomposition (OID), proposed by Schlegel et al., ${ }^{29}$ which allows decomposition of a flow into either high-energy or high-gain modes that map linearly to some observable of interest (the acoustic field for instance). In the OID framework the notions of high-gain or high-energy modes are associated with the dependent variables only (rather than the non-linear term of the Navier-Stokes equations), and the notion of linear mapping is based on correlations between the flow modes and the observable. These issues make physical interpretation of OID modes problematic and it is in this that the ERM decomposition proposed here is fundamentally different. Because the modal decomposition is formulated in the framework of the system described by equation (7), the notions of high-gain or high-energy modes have a firmer physical basis, and permit clearer physical interpretation.

The explicit inclusion and decomposition of the non-linear term associated with a given mean flow is in itself an originality of the current work; but the real beauty of the approach is the associated, simultaneous, decomposition of the dynamical system described by equation (7). As this system describes the dynamics of forced linear wavepackets and their sound radiation, its decomposition with respect to different objectives allows us to answer specific questions regarding those dynamics; this is something the OID and other decomposition frameworks are not equipped to do.

In what follows, we use a mnemonic in order to compactly refer to the different modal decompositions using different output mappings. In particular we refer to modes

$$
\text { XXX-Z-\#-v }
$$

where XXX refers to the decomposition (POD or ERM), and Z refers to the output quantity determined by $C$, i.e. $\mathrm{Z}=I, P$, or $M$. When needed, the \# refers to the mode number, and v refers to the flow quantity 
being displayed, i.e. $\mathrm{v}$ takes one of the values $u_{x}, u_{r}, u_{\theta}, \nu$ or $p$, when we are plotting states, or one of the values $f_{u_{x}}, f_{u_{r}}, f_{u_{\theta}}, f_{\nu}$ or $f_{p}$ when we are plotting the corresponding forcing fields in the streamwise, radial, or azimuthal momentum equations, continuity and energy equations, respectively.

\section{Results}

Figure 2 shows cumulative reconstructions of the output for each of the decompositions considered for three different frequencies and $m=0$. If the turbulence were completely random, we would expect the energy to accumulate linearly with the number of modes in the reconstruction. Such a linear trend line has been drawn as a guide on Figure 2.
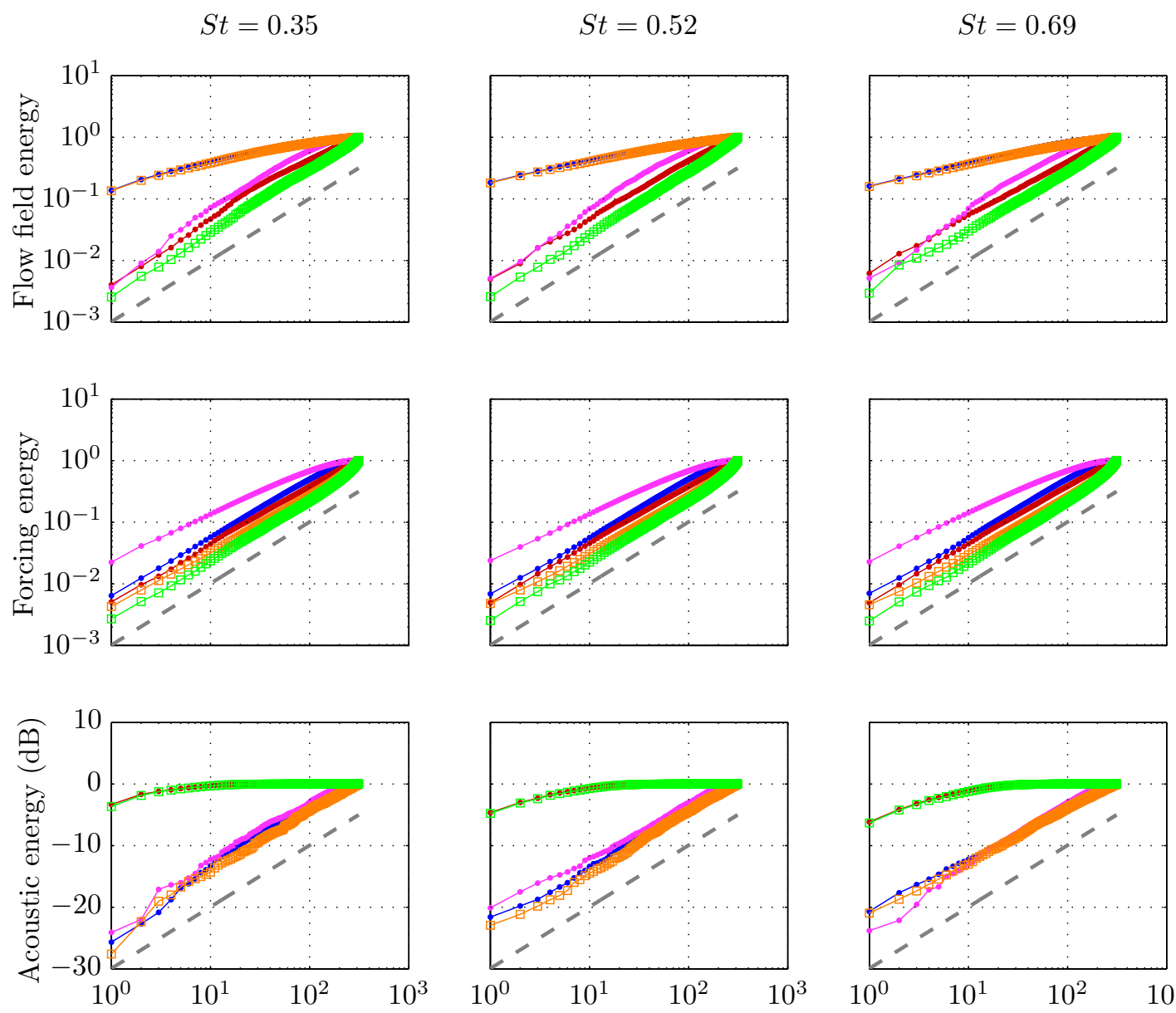

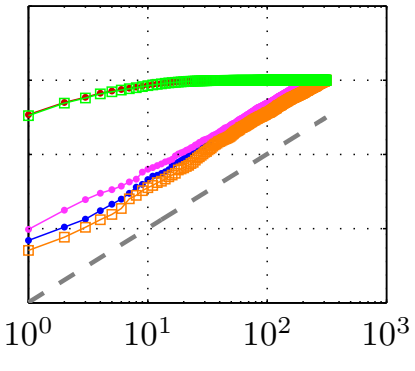

Number of modes

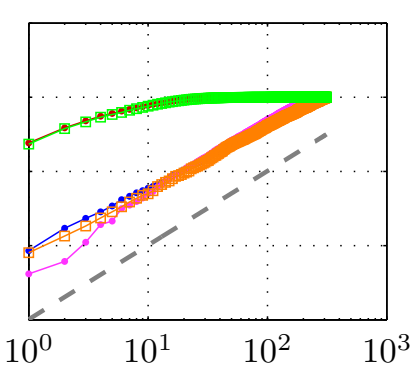

Number of modes

Figure 2: Cumulative near-field, forcing, and far-field energy captured by a reduced-order construction of the LES data using a varying number of the following types of modes: - POD-I; - POD-P; POD-M; $\square$ ERM-I; $\square$ ERM-P. The gray dashed line shows linear accumulation of energy, indicating an equal division of energy between all modes of a decomposition.

Recall that POD modes, by definition, represent the maximal portion of the output energy that can be achieved by any reconstruction of that order. This can be observed in Figure 2 by noting that the PODI curve is always highest in the flow field reconstruction (first row), the POD-M curve is always highest in the forcing field reconstruction (second row), and POD-P curve is always highest in the acoustic field reconstruction (third row).

The first few POD-I modes (blue lines with circles) comprise a significant fraction of the total flow energy, indicating that coherent structures are significant at each of the frequencies considered. These modes take 
the form of wavepackets; their structure will be examined in the next section. Despite the high energy of these structures in the flow field, they comprise (are correlated with) a minuscule amount of the acoustic energy. The near linear slopes of the POD-I acoustic field reconstructions show that these modes are no more effective at capturing the acoustic energy than a random representation of the flow field. For each frequency, the first three POD-I modes, which cumulatively represent over $50 \%$ of the flow field energy, produce an acoustic field that is too quiet by about $20 \mathrm{~dB}$.

Likewise, when POD-P is considered (red curves with circles), the first three modes reproduce the acoustic field to within $2 \mathrm{~dB}$ but represent a vanishing fraction of the flow field energy. These observations are similar to those made in a previous POD analysis of a low Reynolds number jet. ${ }^{26}$ The interpretation is that both the near-field and far-field contain very energetic coherent structures, but they are not mutually correlated.

The ERM-I modes are nearly as efficient in reconstructing the flow field as the POD-I modes (compare orange and blue curves). Similarly, the ERM-P modes are nearly as efficient in reconstructing the acoustic field as the POD-P modes (compare red and green curves). In other words, in both cases, the modes with the highest gain are also most prevalent in the data. As discussed at the end of section III.C, this suggests that the forces contain little underlying structure capable of preferentially forcing certain modes. The close relationship between the leading ERM and POD modes will be further highlighted by examining their flow and forcing fields in the next section.

The POD-M modes further highlight the lack of underlying structure in the forcing field. The most energetic POD-M modes make up only a small fraction of the total forcing energy, signifying a lack of energetic coherent structures. The POD-M modes are also extremely inefficient at describing the energetic structures in the flow field and acoustic field. This indicates that the coherence structures that do exist in the forcing field are not responsible for exciting the coherent structures in either the flow field or acoustic field. In fact, they are no more effective at producing these fields than random forces, since the POD-M reconstructions linearly accumulate energy in the flow field and acoustic field.

Conversely, the POD-I, POD-P, ERM-I, and ERM-P decomposition are all extremely inefficient at describing the forcing field. Again, each reconstruction is nearly linear with a slope of one, showing that the forces responsible for exciting these modes are nearly random.

The gains associated with the ERM modes are shown in Figure 3, and are typical of those obtained from non-normal operators. Detailed comparisons with non-empirical resolvent modes will be made in future publications.
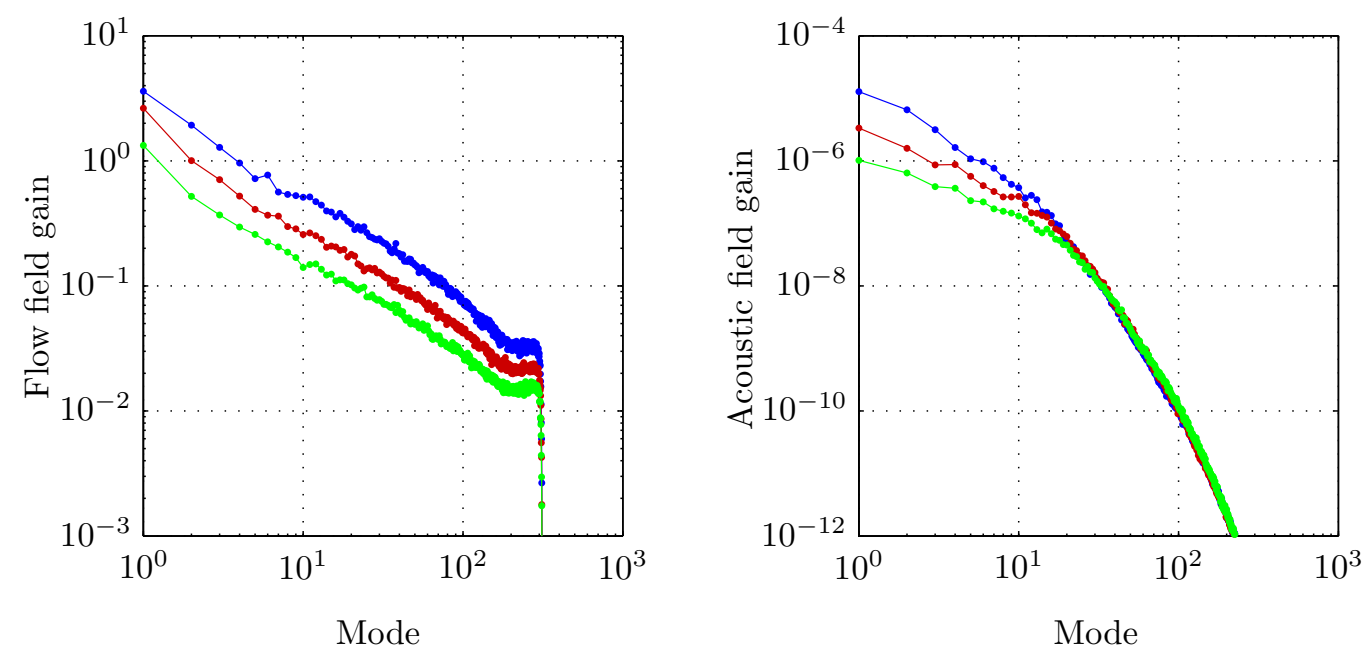

Figure 3: Gain between the forcing and flow field (ERM-I) and forcing and acoustic field (ERM-P) for each frequency: $\bullet S t=0.35 ; \bullet S t=0.52 ; \bullet S t=0.69$. 


\section{Modal structure: flow field decompositions}

In this section, we describe the structure of the various modes and attempt to relate them to the underlying dynamics of the near-field turbulence and far-field acoustics. We have examined a large number of the individual modes, but we limit the discussion to what we believe are the most salient features. Indeed, for each frequency, decomposition, and mode, there are 5 flow variables and 5 forces that can be plotted (a total of 46800 possible plots!). With few exceptions, we limit the field plots to pressure and axial velocity and their corresponding forces in the energy and axial momentum equations. The pressure is largely representative of the specific volume (the structures are nearly isentropic), and there are only minor differences in the forces we observe in the axial, radial, and azimuthal momentum equations. Finally, the conclusions are similar for all frequencies considered (up to $S t=1$ ), so for the following two sections we focus on the results for $S t=0.35$, representative of the most energetic frequency in the near field.

Moreover, each mode is complex-valued. Again, to streamline the discussion and minimize the number of plots, we use the following scheme. For modes that are observed to propagate as coherent waves, we plot the real part, noting that the imaginary part has a similar structure but phase shifted by 90 degrees. Likewise, as the amplitude of each mode is arbitrary, we scale each one to unit amplitude and plot them on a linear scale.

On the other hand, we do not observe the forces to be globally coherently advecting disturbances (a fact which is significant in their interpretation). We therefore plot their amplitudes, scaled by their norm, using logarithmic contours so that regions of comparatively smaller forcing can be viewed alongside those regions with higher forcing.

Figure 4 depicts the structure of the pressure field for the first POD-I mode (POD-I-1-p), which is similar to many previous observations of coherently advecting, near-field wavepackets. Also depicted in the figure is the pressure field obtained by forcing the linearized Euler equations exclusively at the inlet using a KelvinHelmholtz eigenfunction (no volumetric forcing), solved using the one-way Euler method. ${ }^{30}$ This model solution provides a reasonably close representation of the POD mode to well past the close of the potential core, as has been noted in previous investigations.

However, on close examination, there is an important difference in the two structures: the POD mode has a slight phase shift in the radial direction at around the lip line and moving towards the centerline near the close of the potential core. We believe this phase shift is indicative of distributed forcing of the wavepacket. This hypothesis is supported by the spatial distribution of the forces, which will be examined later.
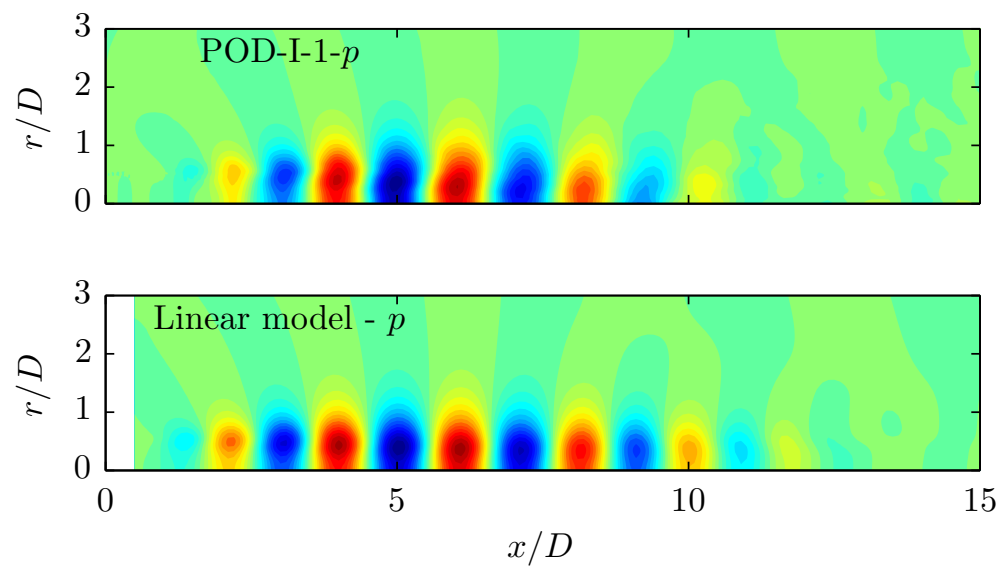

Figure 4: The pressure field for POD-I-1 and from a model solution of the linearized equations under inlet forcing.

Figure 5 shows the flow field structure of the first, second, and fortieth POD-I and ERM-I modes. The POD-I and ERM-I representations of the first two modes are nearly identical, and we have already seen that their contribution to the flow field energy is also nearly equal. This further establishes our previous conclusion - that the energetic flow structures are excited by incoherent forces. By contrast, the fortieth mode in each decomposition captures low energy turbulent behavior. The associated POD-I and ERM-I 

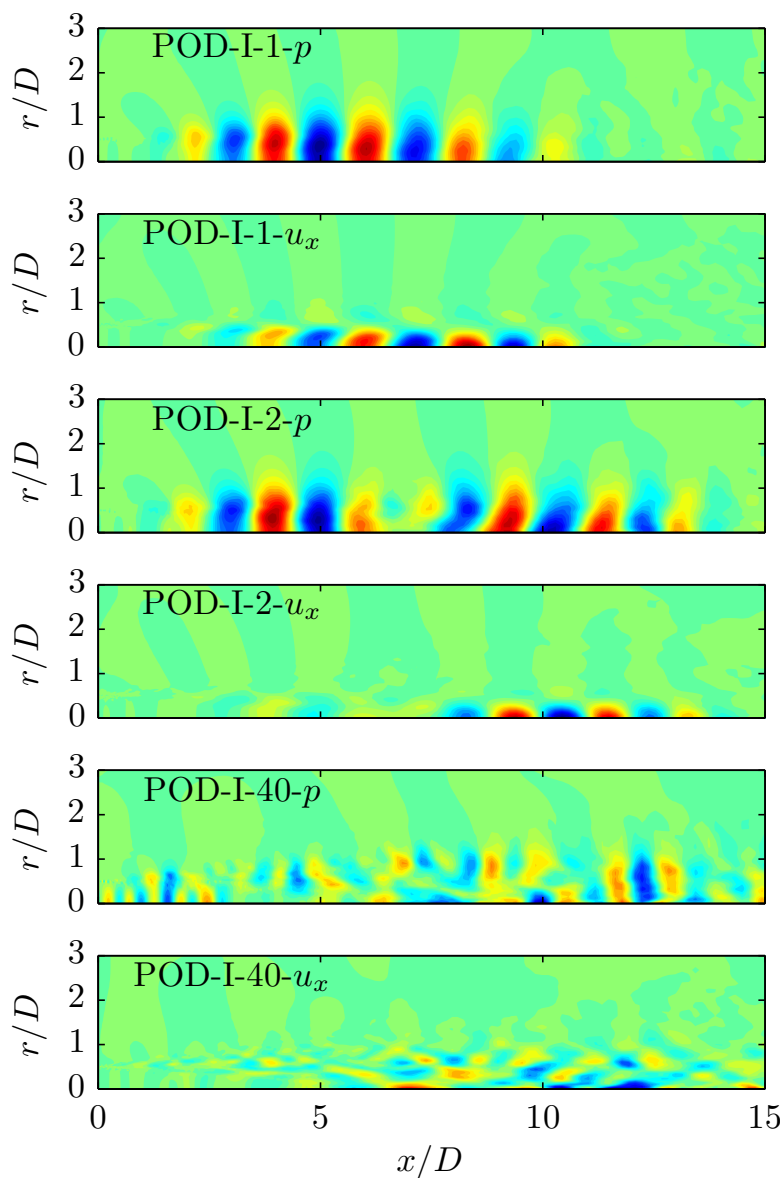
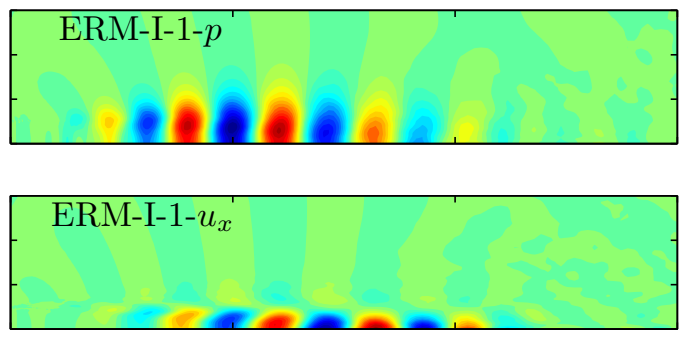

ERM-I-2-p
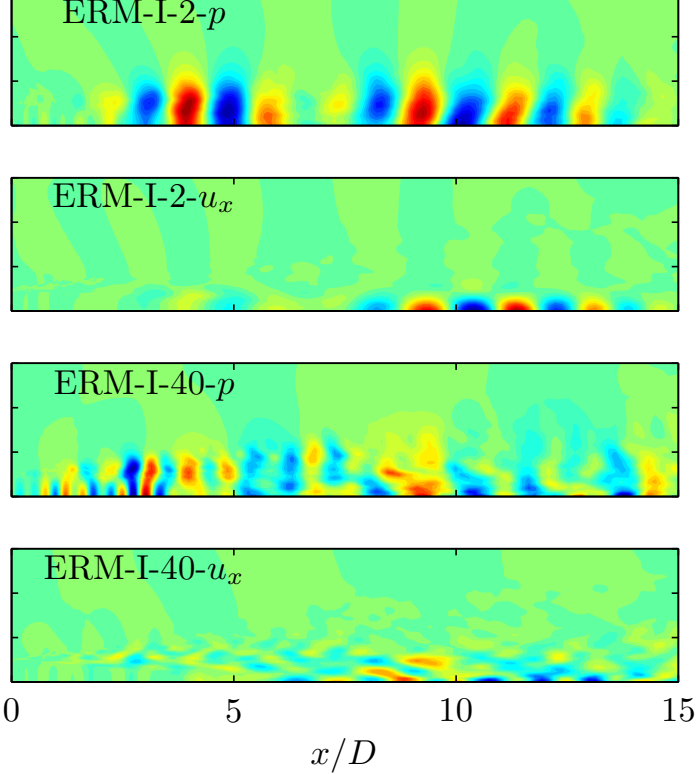

Figure 5: Comparison for POD-I and ERM-I modes at $S t=0.35$. The pressure and axial velocity are shown for the first, second, and fortieth modes of each decomposition. The close correspondence of the first two modes highlight the equivalence of high energy and high gain modes in the flow field.

modes are not identical, presumably because many modes exist with similar energies and gains.

The $f_{p}$ and $f_{u_{x}}$ components of the forces associated with these modes are shown in Figure 6 . The structure of the forces is strikingly different from that of the flow field wavepackets themselves. The forces are very concentrated in a thin layer starting in the shear layer near the nozzle exit and approximately following a contour of constant mean axial velocity as we move downstream. Moreover, as noted above, the forcing does not reveal a clear or coherent phase pattern: it is not propagating with the corresponding wavepacket. The forces associated with the coherent flow structures (modes 1 and 2) are nearly identical to those associated with the unstructured turbulence (mode 40), indicating that the same forcing mechanisms are responsible for both.

The $f_{p}$ components are especially large in the near-nozzle region and comparatively small elsewhere. This near-nozzle forcing could be related to the upstream excitation of the Kelvin-Helmholtz instability. Although not shown, the $f_{\nu}$ component is nearly identical to the $f_{p}$ component, indicating that the forcing is nearly isentropic. The $f_{u_{x}}$ components are somewhat more distributed, having significant magnitude throughout the shear layer and beyond the close of the potential core. As noted, the strongest forces in the shear layer approximately follow a line of constant axial velocity. Since the near-field wavepackets represented by these modes are know to convect with nearly constant phase velocity, lines of constant velocity approximate the location of the critical layer, a location at which the jet has been shown to be sensitive to forcing. ${ }^{31}$ Figure 7 shows ERM-M-1- $f_{p}$ overlaid by contours of axial velocity at Mach 0.6 and Mach 0.8 , which provide a reasonable bound on the possible phase speeds of the coherent structures. This estimated region nicely 

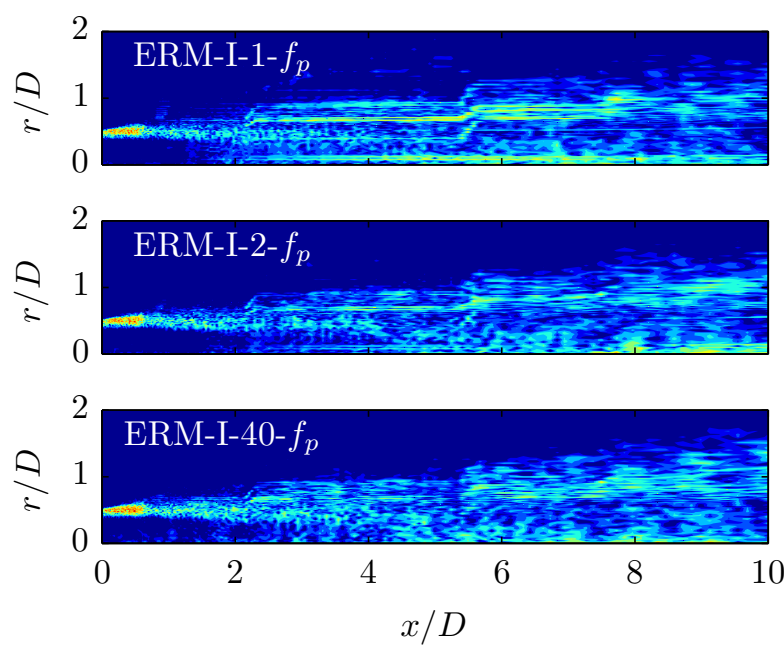
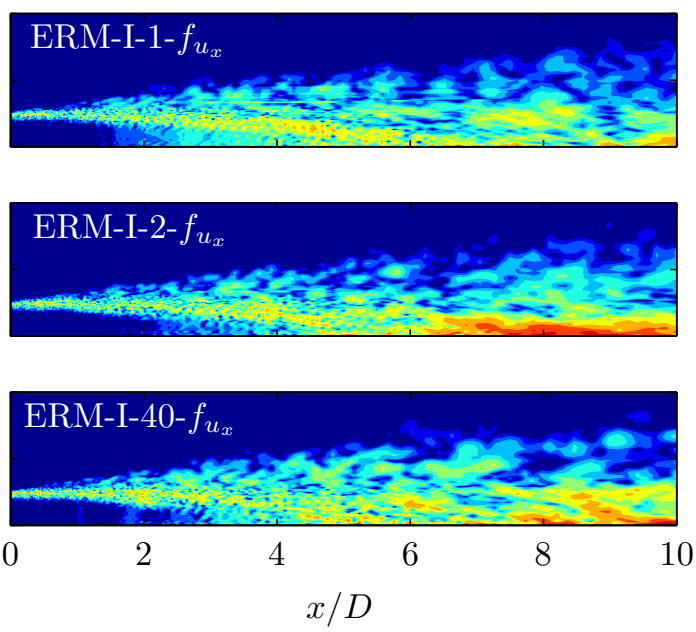

Figure 6: Forces associated with the first, second, and fortieth ERM-I modes at $S t=0.35$. The forces that excite both high and low gain modes are structurally similar, and their form is suggestive of random turbulence.

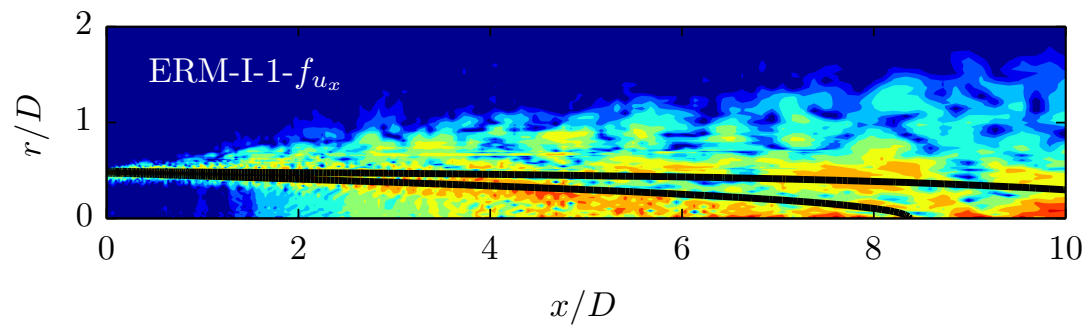

Figure 7: Axial momentum forcing of ERM-I-1, overlaid by lines of constant mean axial velocity corresponding to Mach 0.6 and 0.8. These lines are meant to approximate the location of the critical layer, and the forces are concentrated in this region.

predicts the spatial distribution of the momentum forcing up to the end of the potential core.

Unlike the near-nozzle and shear layer forces, the momentum forces beyond the end of the potential core show some phase structure indicative of coherent convection. The real part of ERM-I-1- $f_{u_{x}}$ is shown in Figure 8 as an example. The form is suggestive of nonlinear interaction between two low frequency structures, possibly due to nonlinear saturation and breakdown of coherent structures that is known to occur in this region of the jet. These forcing structures are less prevalent in the POD-P and ERM-P modes (shown later), indicating limited acoustic significance, consistent with the fact that little noise emanates from this region of the jet.

At higher frequencies, the axial momentum forces associated with the first few POD-I and ERM-I modes exhibit phase structure that may indicate nonlinear wavepacket interactions. For example, Figure 9 shows the real part of three ERM-I modes at $S t=0.69$. We will see shortly, however, that these interactions do not appear to be related to sound generation.

A concern moving forward is the lack of spatial structure and noisiness of the associated forcing fields. At this stage we are uncertain as to whether this reflects a lack of statistical convergence or a genuinely smallscale process. As noted above, the decompositions were computed with differing numbers of realizations with only minor variations in their spectrum and spatial structure, and it was also verified that the spatial noisiness is not associated with aliasing from differentiating the LES data. The forces by definition are a convolution of all frequencies and azimuthal modes that sum to the chosen $S t=0.35$ and $m=0$, and, 

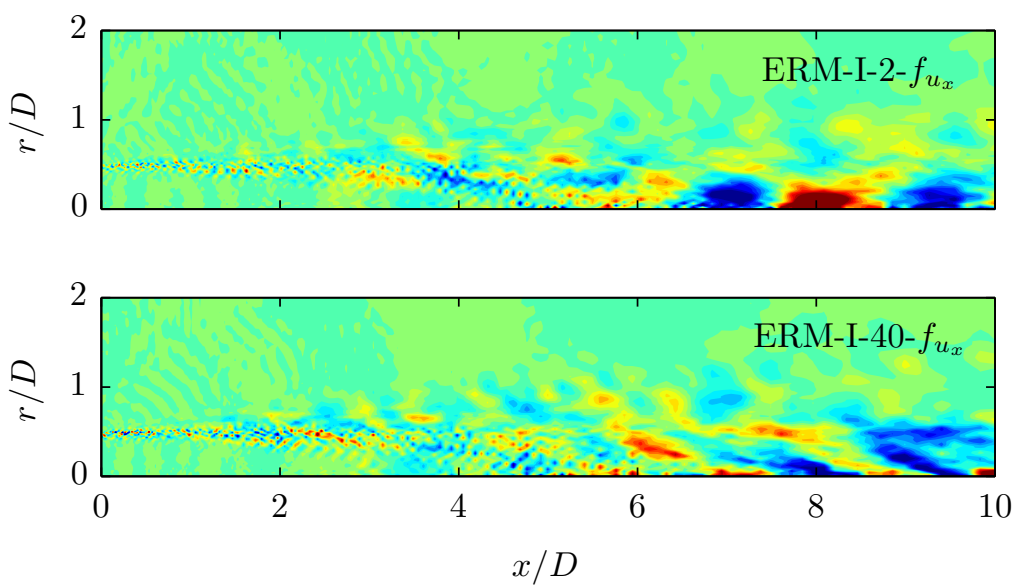

Figure 8: The phase structure of the forces at $S t=0.35$ show that they do not propagate coherently, except downstream of the end of the potential core.
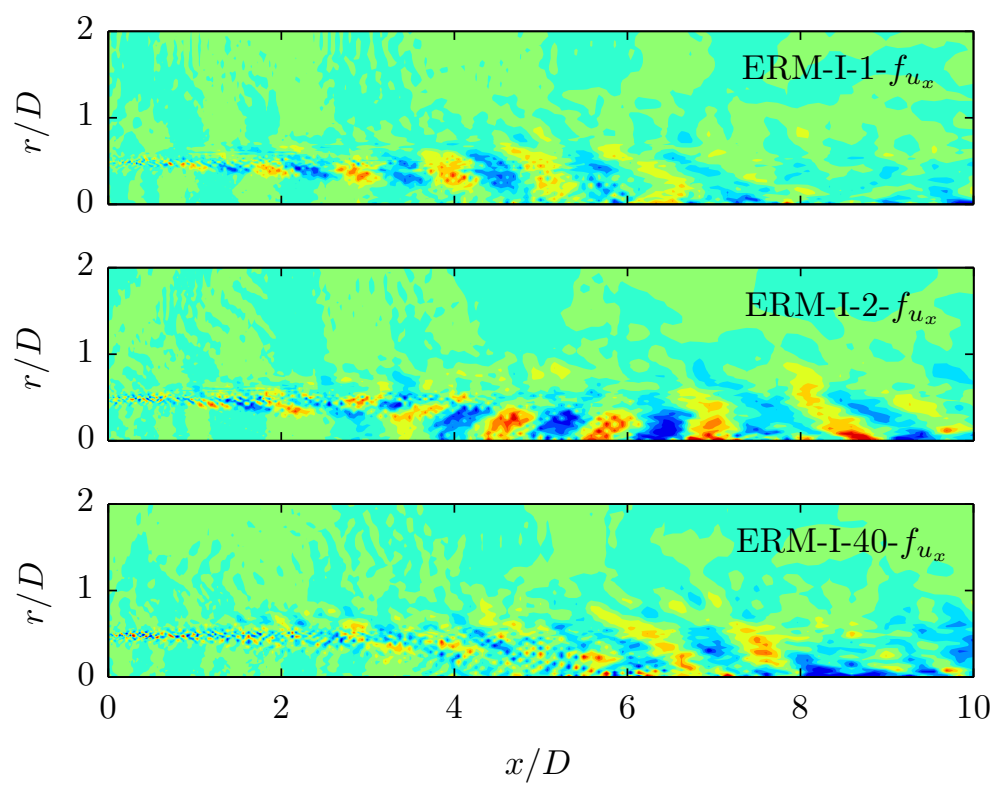

Figure 9: The phase structure of the ERM-I axial momentum forces at $S t=0.69$ shows some evidence of nonlinear wavepacket interactions.

without breaking them apart into their individual frequencies and wavenumbers we cannot say much about what scales generate them. At this stage, we can only say that the process is largely incoherent, meaning that they are unlikely to have been generated by nonlinear interactions amongst coherent structures.

\section{IV.A. Modal structure: acoustic field decompositions}

Figure 10 shows the flow field and acoustic field structure of the first two POD-P and ERM-P modes. In the first mode of both decompositions, a single, highly directive beam of acoustic radiation emanates from near the close of the potential core, accounting for over half of the radiated acoustic energy of the jet. The near flow field takes the form of a distorted wavepacket. 

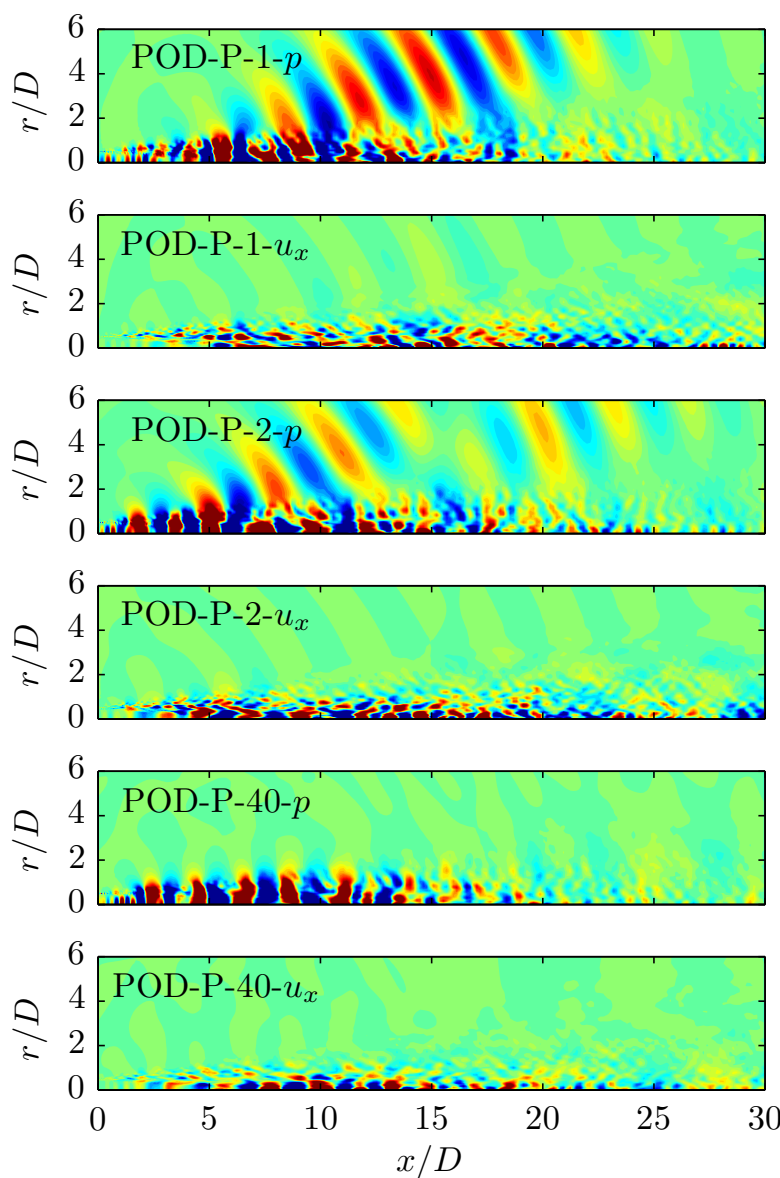
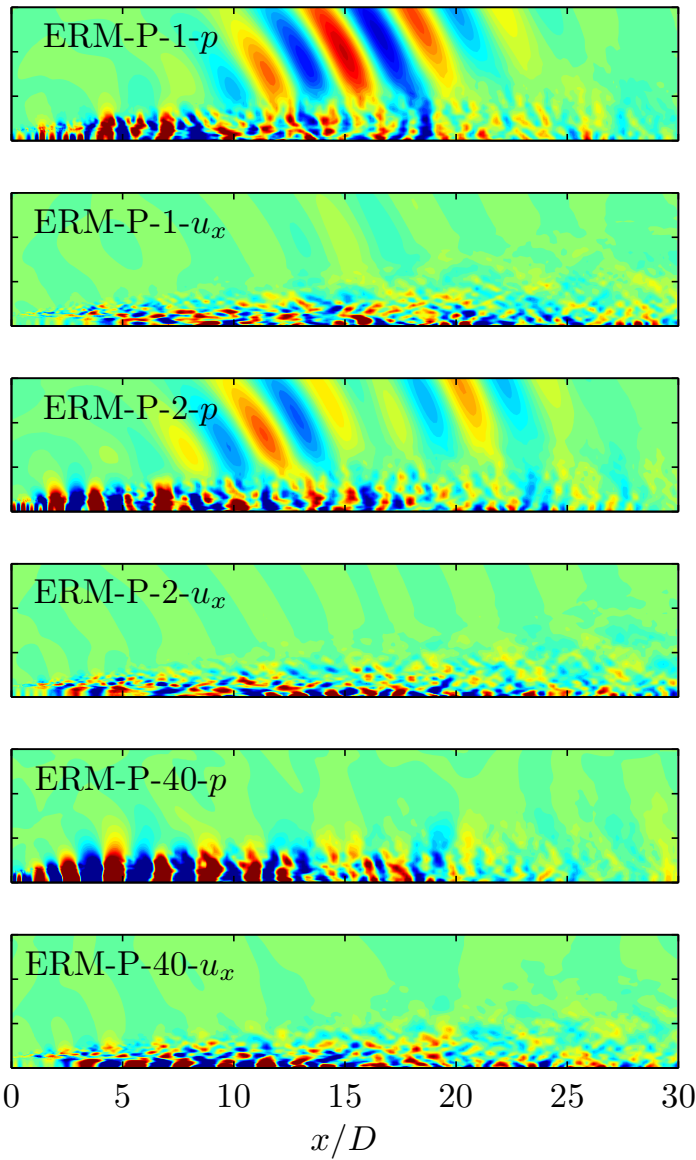

Figure 10: Comparison of POD-P and ERM-P modes at $S t=0.35$. The pressure and axial velocity are shown for the first, second, and fortieth modes of each decomposition. The close correspondence of the first two modes highlight the equivalence of high energy and high gain modes in the acoustic field.

Overall, the POD and ERM modes are again similar to each other, although slightly larger differences can be detected than were observed for the POD-I and POD-P decompositions. Also recall that both modes make an almost identical contribution to the acoustic field energy. The similarity between the two types of modes again confirms that the forcing is essentially incoherent. The minor differences are likely due to lack of convergence caused by the relatively small number of jet realizations. The acoustic decompositions converge more slowly than the flow field decompositions since the high amplitude noise events they are attempting to distill are intermittent and therefore exist in only a fraction of the realizations.

The corresponding forces that produce the POD-P and ERM-P modes are shown in Figure 11. They follow the same general trends as the high-gain forces to the flow field, again reflecting a lack of coherence. This is, emphatically, not equivalent to saying that the random forces excite random acoustic waves. The acoustic field (at this low frequency) is very coherent and, indeed, the modes in Figure 10 show that the acoustic field and the corresponding flow field have the spatial appearance of pieces of wavepackets, attaining their maximal energy near the close of the potential core. Our preliminary interpretation is that both the energetic nearfield structures and the energetic acoustic waves are generated as linear solutions of equation (7) after having been excited by incoherent turbulent fluctuations. A corollary is that the acoustic field is not associated with nonlinear interactions amongst coherent structures, as has been sometimes suggested in the past. While further analysis is required to state firm conclusions, the present results bode well for the construction of accurate linear models that capture the energetic, coherent fluctuations in both the flow and acoustic field.

The mode and force structures at higher frequencies are similar to those so far discussed for $S t=0.35$. 

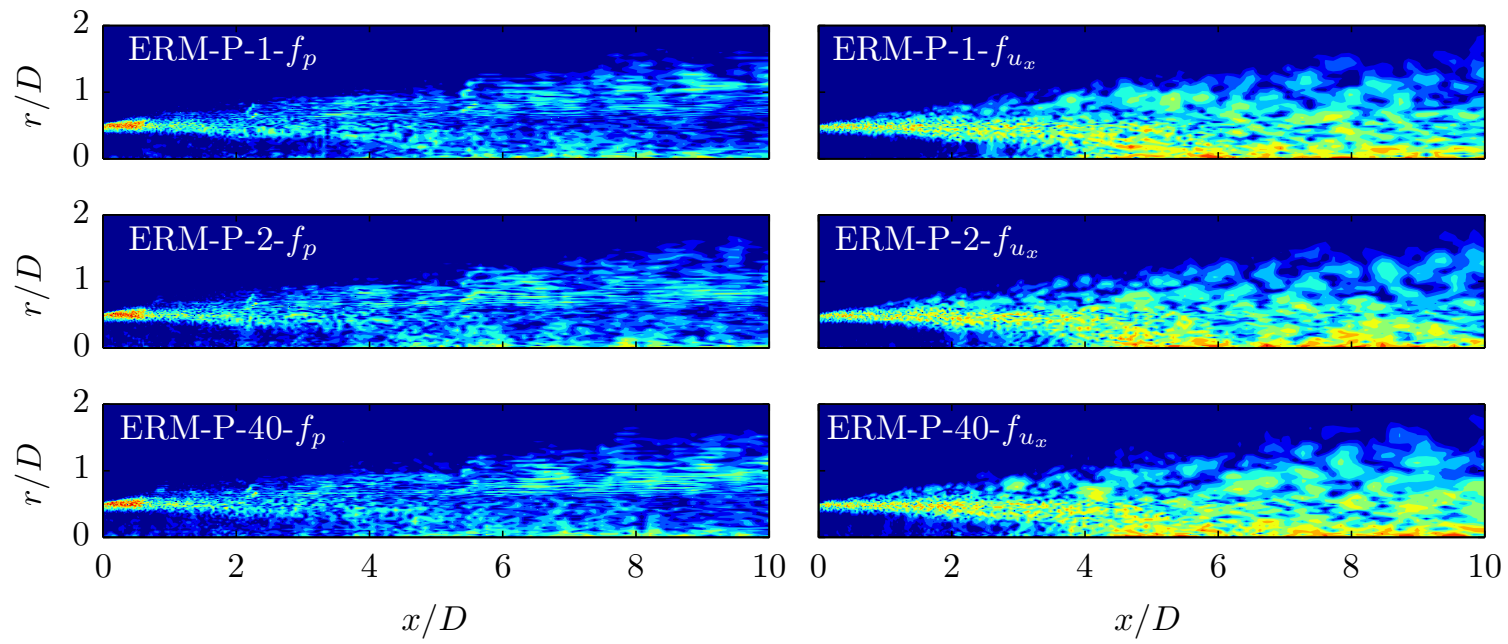

Figure 11: Forces associated with the first, second, and fortieth ERM-P modes at $S t=0.35$. The forces that excite both high and low gain modes are structurally similar, and their form is suggestive of random turbulence.

As an example, Figure 12 shows POD-P and ERM-P modes and Figures 13 and 14 show the corresponding ERM-P forces for $S t=0.69$. Unlike the flow field forces, the acoustic field forces do not display any additional phase structure at higher frequencies, indicating that the possible nonlinear wavepacket interactions observed in the flow field are not correlated with acoustic radiation.

\section{Summary}

In this paper, we have used LES data from a Mach 0.9 turbulent jet to examine the role and character of nonlinear forcing on hydrodynamic wavepackets and their acoustic radiation. The linearized Navier-Stokes operator was used to calculate the nonlinear forcing terms associated with independent ensembles of the LES data. To reveal the structure within the flow and force data ensembles, empirical techniques were employed to extract several types of modes. Modes with high energy in either the flow field or acoustic field were extracted using proper orthogonal decomposition. Consistent with previous investigations, the modes with high energy in the flow field have low energy in the acoustic field and vice versa.

Modes with high gain either between the forces and the flow field or between the forces and the acoustic field were extracted using a novel empirical resolvent mode technique (ERM). The high gain modes are found to be similar to the corresponding high energy modes, suggesting that the coherent structures in both the flow field and acoustic field are excited by forces that contain little structure and are instead nearly random.

Likewise, the spatial structure of these forces is different from that of the flow field and acoustic field wavepackets themselves. They are concentrated in the shear layer, particularly near the critical layer, and do not, for the most part, propagate along with the wavepackets. This suggests that these forces correspond primarily to incoherent turbulent fluctuations rather than nonlinear interactions between coherent structures. The same result is seen for both the forces that maximize gain to the flow field and those that maximize gain to the acoustic field, despite the fact that the fields themselves are dominated by coherent structures. It appears that turbulent fluctuations excite and "jitter" wavepackets that radiate acoustic energy.

We have thus far only analyzed the axisymmetric mode of the flow field, and it remains to be verified that these results hold at other acoustically relevant azimuthal wavenumbers. With this caveat, the findings of this paper suggest that linear models that capture both the coherent flow and acoustic fields can be constructed if appropriate parameterizations of the stochastic forcing can be found, since such forcings will excite the high gain modes to produce the observed coherent structures in both the near- and far-field. 

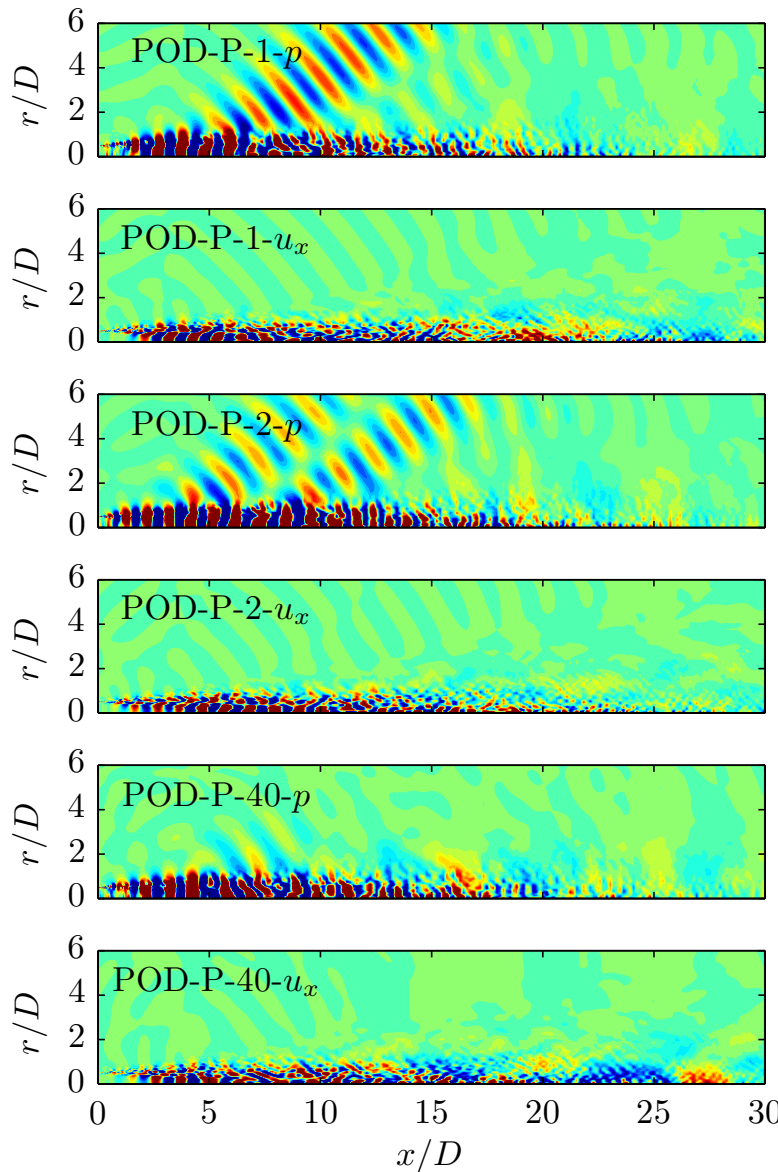
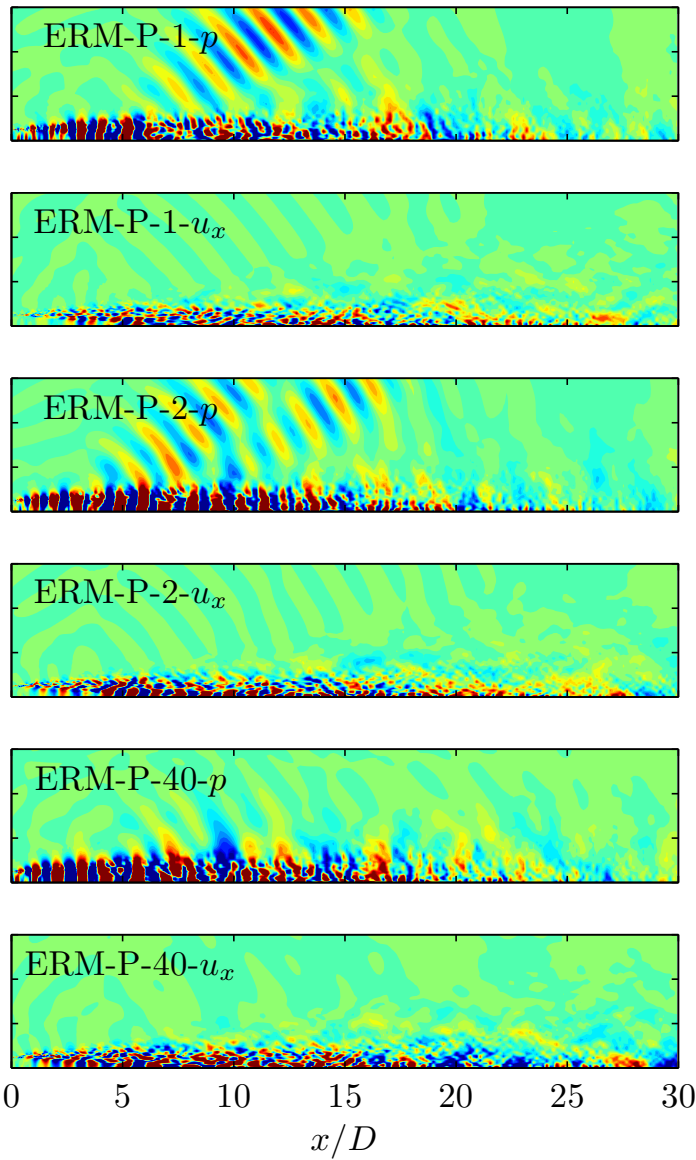

Figure 12: Comparison of POD-P and ERM-P modes at $S t=0.69$. The pressure and axial velocity are shown for the first, second, and fortieth modes of each decomposition.

\section{Acknowledgments}

We are grateful for support from the Center for Turbulence Research at Stanford University where a preliminary version of this analysis was performed during the 2014 Summer Program. The LES study was supported by NAVAIR SBIR project, under the supervision of Dr. John T. Spyropoulos. The main LES calculations were carried out on CRAY XE6 machines at DoD HPC facilities in ERDC DSRC. TC and AT acknowledge earlier support of our jet noise work by the Office of Naval Research under contract N0014-111-0753. We would also like to thank Profs. Sanjiva Lele and Joseph Nichols for helpful discussions on this research.

\section{References}

${ }^{1}$ Jordan, P. and Colonius, T., "Wave Packets and Turbulent Jet Noise," Annual Review of Fluid Mechanics, Vol. 45, 2013, pp. $173-195$

${ }^{2}$ Michalke, A., "An Expansion Scheme for the Noise from Circular Jets," Z.. Flugwiss, Vol. 19, 1971.

${ }^{3}$ Mattingly, G. E. and Chang, C. C., "Unstable waves on an axisymmetric jet column," Journal of Fluid Mechanics, Vol. 65, 9 1974, pp. 541-560.

${ }^{4}$ Crighton, D. G. and Gaster, M., "Stability of slowly diverging jet flow," Journal of Fluid Mechanics, Vol. 77, 1976, pp. 397-413.

${ }^{5}$ Tam, C. K. W. and Burton, D. E., "Sound generated by instability waves of supersonic flows. Part 2. Axisymmetric jets," J. Fluid Mech., Vol. 138, 1984, pp. 273-295. 

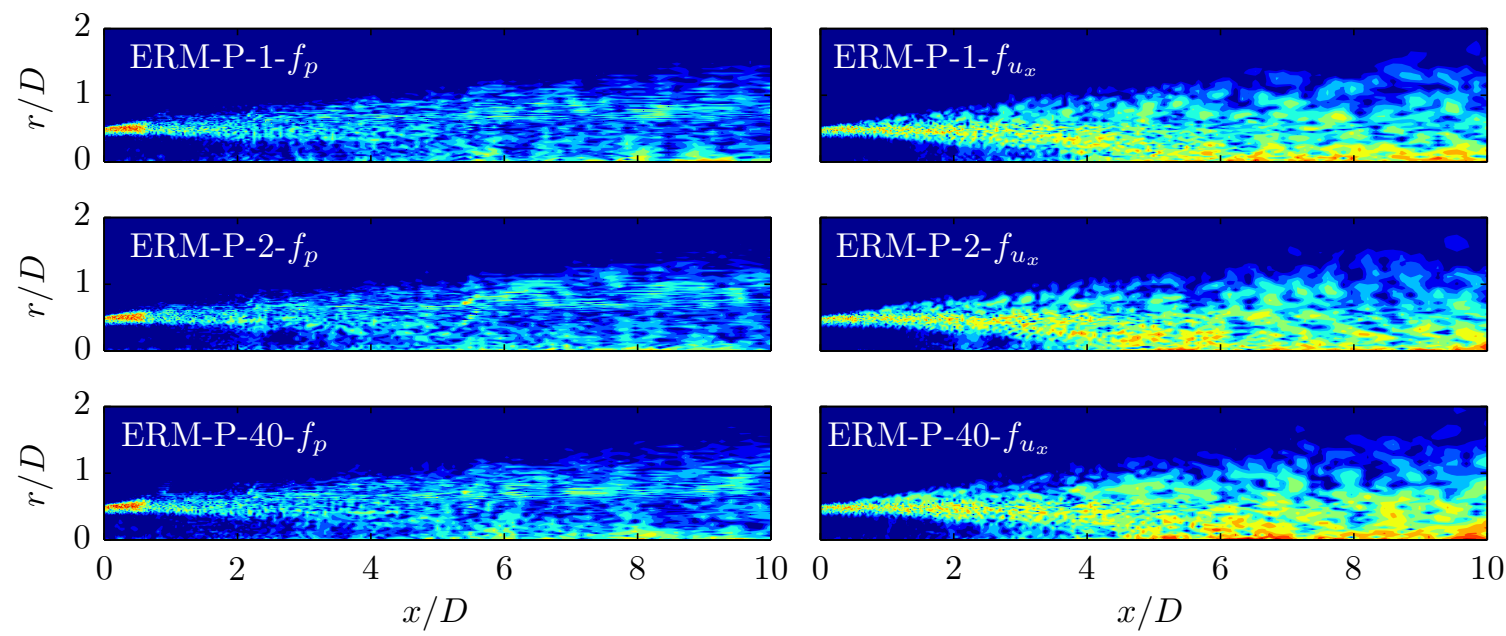

Figure 13: Magnitude of the forces associated with the first, second, and fortieth ERM-P modes at $S t=0.69$.

${ }^{6}$ Gudmundsson, K. and Colonius, T., "Instability wave models for the near-field fluctuations of turbulent jets," J. Fluid Mech., Vol. 689, 2011, pp. 97-128.

${ }^{7}$ Sinha, A., Rodriguez, D., Bres, G., and Colonius, T., "Wavepacket models for supersonic jet noise," J. Fluid Mech., Vol. 742, 2014, pp. 71-95.

${ }^{8}$ Nichols, J. and Lele, S., "Global modes and transient response of a cold supersonic jet," J. Fluid Mech., Vol. 669, 2011, pp. 225-241.

${ }^{9}$ Baqui, Y. B., Agarwal, A., Cavalieri, A., and Sinayoko, S., "Nonlinear and linear noise source mechanisms in subsonic jets," 19th AIAA/CEAS Aeroacoustics Conference, AIAA Paper 2013-2279, Berlin, Germany, May 2013.

${ }^{10}$ Jordan, P., Colonius, T., Bres, G. A., Zhang, M., Towne, A., and Lele, S., "Modeling intermittent wavepackets and their radiated sound in a turbulent jet," Tech. rep., Proceedings of the Center for Turbulence Research summer program, 2014.

${ }^{11}$ Cavalieri, A. V. G., Jordan, P., Agarwal, A., and Gervais, Y., "Jittering wave-packet models for subsonic jet noise," Journal of Sound and Vibration, Vol. 330, No. 18, 2011, pp. 4474-4492.

${ }^{12}$ Kerhervé, F., J. P. C. A. V. G. D. J. B. C. and Juvé, D., "Educing the source mechanism associated with downstream radiation in subsonic jets," Journal of Fluid Mechanics, Vol. 710, 2012, pp. 606.

${ }^{13}$ Zhang, M., Jordan, P., Lehnasch, G., Cavalieri, A., and Agarwal, A., "Just enough jitter for jet noise," 20th AIAA/CEAS Aeroacoustics Conference, 2014.

${ }^{14}$ Brès, G. A., Jordan, P., Colonius, T., Rallic, M. L., Jaunet, V., and Lele, S. K., "Large eddy simulation of a turbulent Mach 0.9 jet," Tech. rep., Proceedings of the Center for Turbulence Research summer program, 2014.

${ }^{15}$ Brès, G. A., Jaunet, J., Le Rallic, M., Jordan, P., Colonius, T., and Lele, S. K., "Large eddy simulation for jet noise: the importance of getting the boundary layer right," Submitted to the 21sst AIAA/CEAS Aeroacoustics Conference, 2015.

${ }^{16}$ Ahnert, K. and Abel, M., "Numerical differentiation of experimental data: local versus global methods," Computer Physics Communications, Vol. 177, No. 10, 2007, pp. $764-774$.

${ }^{17}$ Lumley, J. L., "The Structure of Inhomogeneous Turbulent Flows," Atmospheric turbulence and radio propagation, edited by A. M. Yaglom and V. I. Tatarski, Nauka, Moscow, 1967, pp. 166-178.

${ }^{18}$ Sirovich, L., "Turbulence and the Dynamics of Coherent Structures, Part III: Dynamics and Scaling," Quarterly of Appl. Math., Vol. XLV, 1987, pp. 583-590.

${ }^{19}$ Borée, J., "Extended proper orthogonal decomposition: a tool to analyse correlated events in turbulent flows," Experiments in Fluids, Vol. 35, No. 2, 2003, pp. 188-192.

${ }^{20}$ Maurel, S., Borée, J., and Lumley, J., "Extended proper orthogonal decomposition: Application to jet/vortex interaction," Flow, Turbulence and Combustion, Vol. 67, No. 2, 2001, pp. 125-136.

${ }^{21}$ Rowley, C. W., "Model reduction for fluids, using balanced proper orthogonal decomoposition," International Journal of Bifurcation and Chaos (IJBC), Vol. 15, No. 3, 2005, pp. 997-1013.

${ }^{22}$ Bernstein, D. S., Matrix mathematics : theory, facts, and formulas with application to linear systems theory, Princeton University Press, Woodstock, 2005.

${ }^{23}$ Arndt, R. E., Long, D., and Glauser, M., "The proper orthogonal decomposition of pressure fluctuations surrounding a turbulent jet," Journal of Fluid Mechanics, Vol. 340, 1997, pp. 1-33.

${ }^{24}$ Gordeyev, S. and Thomas, F., "Coherent structure in the turbulent planar jet. Part 1. Extraction of proper orthogonal decomposition eigenmodes and their self-similarity," Journal of Fluid Mechanics, Vol. 414, 2000, pp. 145-194.

${ }^{25}$ Citriniti, J. and George, W., "Reconstruction of the global velocity field in the axisymmetric mixing layer utilizing the proper orthogonal decomposition," Journal of Fluid Mechanics, Vol. 418, 2000, pp. 137-166. 

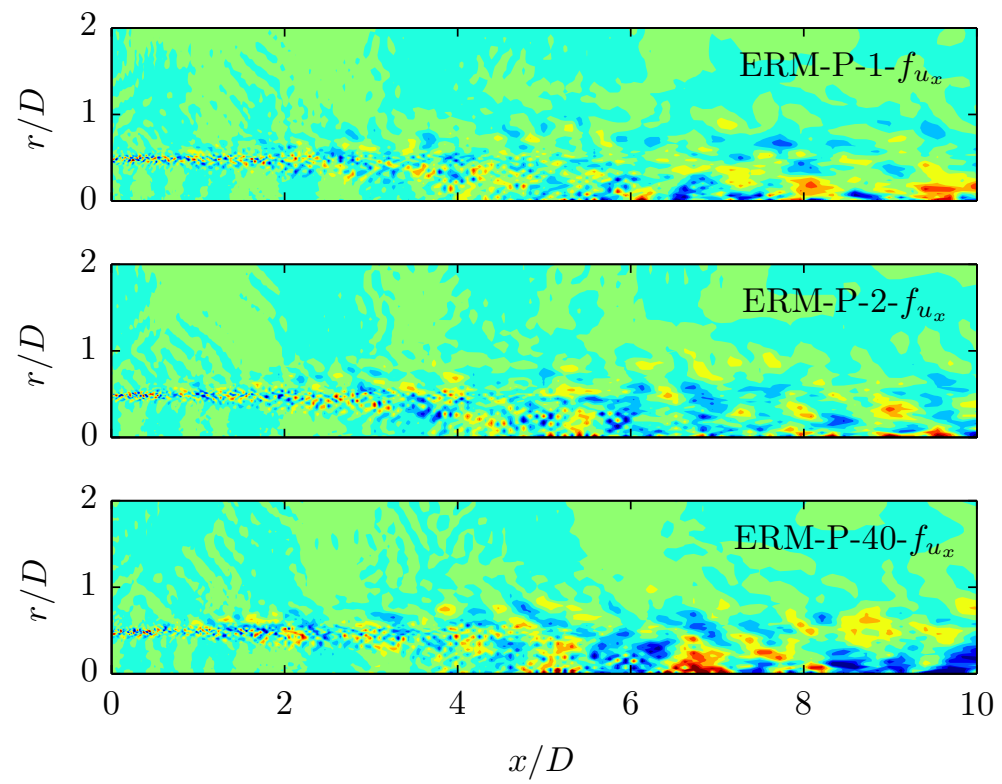

Figure 14: Real part of the axial momentum forces associated with the first, second, and fortieth ERM-P modes at $S t=0.69$. The lack of phase structure is suggestive of random turbulence.

\footnotetext{
${ }^{26}$ Freund, J. and Colonius, T., "Turbulence and sound-field POD analysis of a turbulent jet," International Journal of Aeroacoustics, Vol. 8, No. 4, 2009, pp. 337-354.

${ }^{27}$ Garnaud, X., Lesshafft, L., Schmid, P., and Huerre, P., "The preferred mode of incompressible jets: linear frequency response analysis," Journal of Fluid Mechanics, Vol. 716, 2013, pp. 189-202.

${ }^{28}$ Nichols, J., "none," Tech. rep., Proceedings of the Center for Turbulence Research summer program, 2014.

${ }^{29}$ Schlegel, M., Noack, B. R., Jordan, P., Dillmann, A., Grschel, E., Schrder, W., Wei, M., Freund, J. B., Lehmann, O., , and Tadmor, G., "On least-order flow representations for aerodynamics and aeroacoustics," J. Fluid Mech., Vol. 697, 2012, pp. 367-398.

${ }^{30}$ Towne, A. and Colonius, T., "Continued development of the one-way Euler equations: application to jets," 20th AIAA/CEAS Aeroacoustics Conference, AIAA Paper 2014-2903, Atlanta, Georgia, June 2014.

${ }^{31}$ Tissolt, G., Zhang, M., Lajús, F. C., Cavalieri, A., Jordan, P., and Colonius, T., "Sensitivity of wavepackets in jets to non-linear effects: the role of the critical layer," 21st AIAA/CEAS Aeroacoustics Conference, AIAA Paper 2013-2171, Dallas, Texas, June 2015 .
} 